\title{
Néomutations, âge paternel et syndrome d'Apert
}

La découverte de mutations dans les gènes des récepteurs des facteurs de croissance (FGFR) a d'abord remis en question la classification clinique des craniosténoses [1] en démontrant par exemple qu'une même mutation du gène FGFR2, (localisé sur le chromosome 10 en $10 q 26)$, pouvait aussi bien être à l'origine d'un syndrome d'Apert que d'un syndrome de Pfeiffer. Mais ce n'est pas tout. La connaissance du rôle morphogénétique des FGFR pendant la période embryonnaire chez l'homme devient plus précise grâce à l'analyse du retentissement clinique des différentes mutations sur ces syndromes polymalformatifs. Dans le cas du syndrome d'Apert, qui associe une craniosynostose à des anomalies cérébrales (malformations du corps calleux et des structures limbiques) avec retard mental, ainsi qu'à des syndactylies des mains et des pieds, une fente palatine et diverses autres malformations, on voit à présent que la symptomatologie clinique varie, en partie du moins, selon la mutation: en cas de transversion C934G, qui entraîne la substitution $\operatorname{Ser}^{252} \rightarrow$ Trp, les fentes palatines sont plus fréquentes; en cas de substitution $\mathrm{PrO}^{253} \rightarrow$ Arg, les syndactylies sont nettement plus sévères [2].

\section{L'effet de l'âge paternel}

En outre, l'analyse moléculaire vient de réactualiser l'ancien concept de «l'effet de l'âge paternel ", énoncé pour la première fois par Penrose en 1955 à propos de l'achondroplasie [3]. Il postulait que la probabilité de survenue d'une mutation au cours de la spermatogenèse dans les lignées avec l'âge, en raison du grand nombre de divisions cellulaires se produisant au cours du temps. Comme l'achondroplasie, le syndrome d'Apert, maladie autosomique dominante, est rarement héritée d'un parent malade, mais survient le plus souvent de façon sporadique par néomutation chez un enfant issu de parents indemnes mais plus âgés que la moyenne des couples. On suspectait donc depuis longtemps un effet d'âge paternel dans le syndrome d'Apert, encore que cette hypothèse fût controversée: si l'âge des pères est effectivement augmenté dans les cas de syndrome d'Apert par néomutations, celui des mères l'étant aussi, certains auteurs avaient estimé une origine maternelle dans $30 \%$ des cas [4]. Techniquement, il n'était pas facile jusqu'à présent de trouver l'origine parentale d'une néomutation à l'aide des deux techniques connues: réaliser des hybrides somatiques pour séparer les deux chromosomes 10 d'un malade prend beaucoup de temps; quant à l'analyse des haplotypes des parents et du sujet atteint, elle est possible à condition que celui-ci ait procréé, seul moyen de savoir quel haplotype est lié à la maladie. Cette approche, limitée à un petit nombre de familles, avait cependant permis à Wilkie et à son équipe de montrer que la néomutation était d'origine paternelle dans trois cas [5]. Nouvelle technique pour identifier
l'origine parentale d'une néomutation

La même équipe vient d'adopter une stratégie astucieuse, beaucoup plus efficace et généralisable à l'analyse de toute néomutation [6]. Intitulée
ARMS (amplification refractory mutation system), elle consiste, après avoir repéré des variants normaux proches du site de la mutation, à réaliser en PCR des amplifications sélectives en choisissant les amorces et les enzymes de restriction. La méthode n'est limitée que par l'informativité du polymorphisme de ces variants. Dans le syndrome d'Apert, les mutations se situent toutes près de l'extrémité 5' de l'exon 7 qui comporte $191 \mathrm{pb}$. L'analyse de 1,4 kb de l'intron en amont, et de la totalité de l'intron en aval $(1,15 \mathrm{~kb})$ avec 18 paires d'amorces différentes sur 48 sujets normaux permit de trouver deux variants communs de part et d'autre des mutations: en amont, à la position - 112, le variant 5' - $\mathrm{CAG}^{\mathrm{C}} /{ }_{\mathrm{A}} \mathrm{CTTG}-3$ ', dit $\mathrm{G} / \mathrm{A}$, et en aval, à la position 769 , le variant 5' - ATTTC / ${ }_{\mathrm{A}}$ TGCA-3', dit C/A. Quatre haplotypes sont donc possibles: GA, GC, AA et AC mais en raison de leur répartition dans la population, l'haplotype AA n'a jamais été observé dans cette étude. L'haplotype AC fut retrouvé en excès avec la mutation mais de plus grandes séries sont nécessaires pour pouvoir affirmer un déséquilibre de liaison.

Des conditions optimales d'amplification furent établies pour une amplification sélective en PCR. Pour le variant $G / A$, l'allèle $A$ est amplifié en PCR par les amorces $2^{\mathrm{A}}$ et 10 et pour le variant $\mathrm{C} / \mathrm{A}$, l'allèle $\mathrm{C}$ est amplifié par les amorces 3 et $9^{A}$ (figure 1). La recherche peut se faire par simple génotypage quand le malade est hétérozygote et que l'un au moins des parents est homozygote, ce qui fut le cas dans 48 familles (figure 1). Lorsque les parents étaient double hétérozygotes, grâce à d'autres 


\section{Digestion tenant compte de la mutation}

Deux éventualités :

Parents normaux

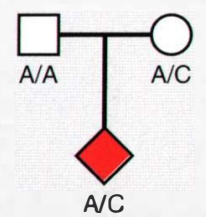

Enfant informatif et atteint du syndrome d'Apert ou bien : coupe avec Sfi l/Bst UI et ne coupe pas avec Bsi Ei/Bgl I

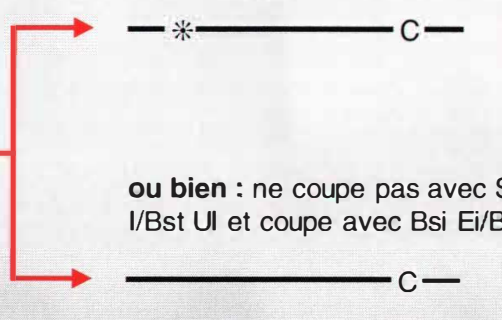

L'allèle $\mathrm{C}$ est donc mutant : la mutation est maternelle l'allèle $C$ du variant $A C$

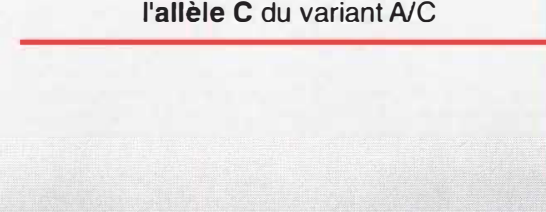

\section{.}

\author{
Bgl I
}

L'allèle $\mathrm{C}$ est donc normal. Par conséquent la mutation est sur l'autre allèle : la mutation est paternelle

Figure 1. Recherche de l'origine parentale par le système ARM. Exemple d'une famille informative pour le polymorphisme C/A (le père étant ici homozygote AA). L'amorce 9A amplifie sélectivement l'allèle C de l'enfant, qui vient obligatoirement de sa mère. La recherche de la mutation est ensuite effectuée sur cet allèle $C$ avec les enzymes de restriction adéquats: si Bsi El/Bgl I coupe et que Sfi I/Bst UI ne coupe pas, cela signifie que I'allèle C est intact et que la mutation dont l'enfant est porteur s'est donc produite dans l'allèle transmis par son père. (D'après [6].)

amorces et d'autres enzymes de restriction, les auteurs parvinrent à déterminer les haplotypes parentaux puis, une fois ceux-ci établis, à retrouver l'origine parentale de la mutation chez les enfants atteints dans 7 familles initialement non informatives. Cela porte donc à 55 le nombre de cas analysés dans cette étude.

\section{Dans le syndrome d'Apert, l'origine} des néomutations est paternelle

Compte tenu de deux cas étudiés précédemment, il est ainsi prouvé que la néomutation est d'origine paternelle dans les 57 cas sporadiques de syndrome d'Apert où celleci fut recherchée. Les âges paternels des familles non informatives étant identiques à ceux des familles informatives, l'hypothèse d'un biais lié à l'informativité peut être éliminée, d'autant plus que les variants choisis avaient la même répartition dans une population témoin.

Ces résultats impressionnants (57 contre 0 ) vont beaucoup plus loin qu'une simple confirmation de l'effet de l'âge parental. Si les cellules germinales mâles se divisent tous les 16jours à partir de la puberté, on conçoit que le fardeau des erreurs mitotiques s'alourdisse avec l'âge.
Mais pourquoi particulièrement dans les FGFR? Un effet d'âge paternel existe en effet dans toutes les maladies dues à des mutations des FGFR, qu'il s'agisse des syndromes d'Apert, de Crouzon, de Pfeiffer, de l'achondroplasie ou du nanisme thanatophore. Récemment dans deux de ces maladies, plusieurs cas de mutations indépendantes chez des germains d'une même famille ont même été rapportés [8]. Les FGFR 1, 2 et 3 seraient donc collectivement prédisposés à muter, de surcroît dans des sites préférentiels. Dans le syndrome d'Apert, les deux mutations prédominantes sont des transversions $\mathrm{C} \rightarrow \mathrm{G}$ survenant dans deux codons adjacents. La mutation $C^{934} \rightarrow G$ est deux fois plus fréquente que la mutation $\mathrm{C}^{957} \rightarrow \mathrm{G}$. Or, elle se trouve dans un contexte CpG et l'on sait que la méthylation de la cytosine est globalement plus importante dans les cellules germinales masculines que dans les cellules germinales féminines. Enfin, mentionnons un élément qui pourrait avoir une importante signification théorique s'il était confirmé: la recherche d'un déséquilibre de liaison entre les haplotypes et la maladie se révéla négative mais, en revanche, elle objectiva une différence de répartition des haplotypes selon le sexe [8]. Or, chez la souris, un déséquilibre de transmission de certains locus autosomiques, co-hérités avec un chromosome $\mathrm{X}$ pourrait aussi être observé en fonction du sexe des descendants.

S.G.

1. Gilgenkrantz S. Les craniosynostoses revues et corrigées par la génétique moléculaire, ou l'irrésistible ascension des récepteurs de facteurs de croissance. médecine/sciences 1995 ; 11 : 1748-51.

2. Slaney SF, Oldridge M, Hurst JA, MorrisKay (MM, Hall (M, Poole MD, Wilkie AOM. Differential effets of FGFR2 mutations on syndactyly and cleft palate in Apert syndrome. Am J Hum Genet 1996; 58: 923-32.

3. Penrose LS. Parental age and mutation. lancet $1955 ; 2: 312-3$.

4. Risch N, Reich EW, Wishnick MM, McCarthy JC; Spontaneous mutations and parental age in humans. Am J Hum Genet 1987 ; 41 : 218-48.

5. Wilkie AOM, Slaney SF, Oldridge M, Poole MI), Ashworth (;.), Hockley AD), Hayward RI), David DJ, Pulleyn I.J, Rutland P, Malcolm S, Winter RM, Reardon W. Apert syndrome results from located mutations of FGFR2 and is allelic with Crouzon syndrome. Nature Genet $1995 ; 9$ : 165-72.

6. Moloney DM, Slaney SF, Oldridge M, Wall SA, Sahlin P, Stemman (; Wilkie AOM. Exclusive paternal origin of new mutations in Apert syndrome. Nature Genet 1996; 13: 48-53.

7. Meyer (;A, Orlow SJ, Mumro IR, Przylepa KA, Jabs EW. Fibroblast growth factor receptor 3 (FGFR3) transmembrane mutation in Crouzon syndrome with acanthosis nigricans. Nature (ienet $1995 ; 9: 462-4$.

8. Sapienza C. A paternal wash in Apert syndrome. Nature Genet $1996 ; 13: 9-10$. 